\title{
Research on Network Teaching Platform-based Personalized Teaching Strategies
}

\author{
Dengjie $^{1, \text { a }}$ \\ ${ }^{1}$ Guangxi Vocational College Of Technology And Business, NO. 15 zhongyao Road, Nannig City, \\ Guangxi, P.R. China \\ a380162982@qq.com
}

Keywords: knowledge representation; teaching evaluation; personalized .

Abstract. According to network teaching characteristics and composition of knowledge structure, discuss the knowledge representation mode of the tree and mesh hybrid structure, analyze this knowledge structured-based teaching evaluation and teaching strategies. In individualized network teaching system, by means of knowledge tree "pruning" and dynamic assessment, it can help learning subjects to understand their current position in their own learning process and mastery situation, by offering appropriate advice and guidance, to achieve self-accumulation and learning functions.

\section{Introduction}

Using the Internet to build distance education system has become a popular new form of learning, and network teaching platform with individualized teaching capabilities is a popular research direction in nowadays. Currently there are various Web-based B / S mode teaching platforms, although they are able to achieve online learning and online testing and evaluation, there are still some problems and shortcomings compared to personalized teaching: (1) online learning cannot provide personalized teaching plans and assessment for different learning subjects; (2) assessment of learning ability of learning subjects is limited to the learning contents of the current stage, lacking of systematic and comprehensive evaluation methods; (3)there are no strong linkages between chapters and sections of teaching materials, weakening intelligence in personalized teaching process. To solve the above problems, adopt artificial intelligence knowledge representation theory, analyze learning package-based curriculum knowledge tree, establish a more systematic and comprehensive evaluation system, so as to maximize individualized teaching.

\section{Tree network knowledge representation-based knowledge representation mode}

Knowledge representation is to use knowledge representation mode that computer can store, identify and process. Whether knowledge representation is reasonable or not directly relates to the smooth conduct of a series of educational activities. Only reasonable knowledge representation and storage, as well as flexible adjustment of knowledge representation mode help to achieve intelligent evaluation and personalized teaching. Reasonable knowledge representation shall meet the following general requirements:

(1) Complete and comprehensive summary and description of knowledge points.

(2) Establishment of relationship between knowledge points is conducive to rapid reasoning.

(3) Reasonably classification of knowledge points.

(4) With module structure beneficial to knowledge base maintenance, updating and efficient search.

In the computer field, the tree structure is used to represent information that has certain association in depth and breadth, there is a "one to many" relationship between data elements, so that knowledge hierarchical relationship, affiliation and parallel relationship can be shown in the tree structure. In most network teaching systems, most of them use tree structure to represent the relationship between curriculum knowledge. Tree structure knowledge representation mode goes from top to bottom, gradually refine knowledge nodes, increases content and complexity layer by 
layer, shrinks extension, this kind of knowledge composition relations conforms to people understand things process in the real world, that is, from simple to complex gradual process.

Use tree structure to represent curriculum knowledge linkage, through traversal process of knowledge tree can achieve curriculum knowledge learning process: preorder traversal starts from leaf nodes and then to root nodes, follow the principle that people are most prone to accept the conventional learning sequence-from easy to difficult; preorder traversal starts from root nodes and then to leaves nodes, to make hard things simple by concept decomposition. No matter traverse in which direction, the tree structure can realize the full curriculum learning process, so the tree structure is the most basic knowledge representation mode in network teaching system.

However, the composition of curriculum knowledge is often not a simple step by step process, which also includes the intricate cross-sections association, such as in the $C$ language, the structural linked list also contains the basic structure of pointers. To maximize the intelligence of teaching strategies, the linkage between internal chapters, and chapters shall be completely represented. In other words, the traditional representation mode of knowledge tree fails to completely represent all the knowledge points of a subject, and therefore cannot well reflect the personalized feature of intelligent teaching platform. For vertical and horizontal spanning associated nodes, mesh structure is the most appropriate means.

For shortcomings of tree structure's simple "one to many" knowledge representation mode, it can use "many to many" network structure to make up. This hybrid representation approach can represent courses' top-down relationship, but also separate "tangled" associated nodes between different chapters, provide comprehensive and complete teaching resource library to realize the greatest degree of personalization in intelligent teaching platform.

\section{Tree-mesh hybrid knowledge representation-based teaching evaluation}

Teaching evaluation is to make value judgment of teaching process and results based on the teaching objectives and serves for teaching activities decision-making. In network teaching platform, the teaching evaluation is a key part, whether it is able to give adaptability evaluation for different learning subject, determines the correctness of teaching strategies. Teaching evaluation generally includes the evaluation of teachers, students, teaching content, teaching methods, teaching environment, teaching management and various factors in the teaching process, but mainly evaluation of student learning outcomes and teachers teaching process. Two core aspects of teaching evaluation: evaluation of teachers teaching work (instructional design, organization, implementation, etc.) - teachers teaching evaluation (in classroom, extracurricular), evaluation of student learning outcomes- exams and testings. Evaluation methods are mainly quantitative evaluation and qualitative evaluation.

Teaching platform always takes teaching objectives as evaluation objectives, converts evaluation objectives into specific, measurable, behavioral and operable standardized system, and then determines the relative importance (weights) of each indicator in constituting a target, finally determines the evaluation index system. For features of network teaching mode, in the preparation of network teaching evaluation index system, it is required to pay attention to the following points: (1) Start from the overall situation. Online teaching takes "learning-centered" instructional design theory as guideline, we should evaluate what knowledge and ability have students mastered through online learning as center. (2) Dynamic. Traditional appraisal theory is basically static summative evaluation, while online teaching evaluation should also attach importance to formative evaluation, namely progressive evaluation, combine evaluation process and teaching process together, collect a variety of assessment information in the teaching process to guide and improve next step teaching. Attach emphasis to dynamic, value its feedback, corrective function is a major feature of network teaching evaluation. (3) Just select typical behavior that can best reflect the evaluation objectives development level of evaluation target, no need to put in all content reflecting the target without missing. For the principle of comprehensiveness to be followed in preparation of index system, it is only required to 
follow main aspects, indicator system only reflects representative typical behavior of evaluation objects, not all behavior.

Based on the above, in the network teaching platform with tree hybrid knowledge representation, to achieve dynamic evaluation and dynamic "pruning" of teaching tree, take learning unit as node package. Node packages are divided into compulsory and optional, identify the degree of difficulty, master degree requirements, pioneer knowledge and subsequent knowledge collection and so on.

\begin{tabular}{|l|l|}
\hline $\begin{array}{l}\text { Attribute } \\
\text { name }\end{array}$ & \multicolumn{1}{|c|}{ Attribute content } \\
\hline ID No. & unique identification number of learning package \\
\hline Description & Description of the concept of knowledge points \\
\hline compulsory & $\begin{array}{l}\text { Knowledge points are divided into course compulsory content and extended } \\
\text { content }\end{array}$ \\
\hline $\begin{array}{l}\text { Weight } \\
\text { number }\end{array}$ & $\begin{array}{l}\text { Dynamically assigned weights, represent comprehensive assessment of node } \\
\text { package }\end{array}$ \\
\hline Pioneer & Pioneer content aggregation of this knowledge point \\
\hline subsequent & subsequent content of this knowledge point \\
\hline Association & $\begin{array}{l}\text { Other associated knowledge points expect pioneer and subsequent content in } \\
\text { the tree structure }\end{array}$ \\
\hline $\begin{array}{l}\text { Degree of } \\
\text { difficulty }\end{array}$ & Values to indicate the degree of difficulty of knowledge points \\
\hline Master & $\begin{array}{l}\text { Knowledge points are categorized into grasp, understand and mastery three } \\
\text { kinds }\end{array}$ \\
\hline
\end{tabular}

Table. 1 Node package content and attribute

Node packages constitute course knowledge tree, and course learning starts from the course tree. After completing learning of each unit and before entering the next unit learning, carry out evaluation of the current unit, and the evaluation results come from online testing, through comprehensive analysis of knowledge points mastery degree, weight, difficulty, learn the knowledge mastery situation of learning subjects, evaluate the next step learning, based on the analysis and evaluation results to decide whether proceed next step learning or new node content, the new node package consolidated weight value is dynamically determined by the evaluation results.

Assume that there are $\mathrm{M}$ knowledge points in node package, $\mathrm{S}$ is the branch weights aggregate, 1 $-\mathrm{n}$ is the retained branches, $\mathrm{k}-\mathrm{m}$ is the trimmed branches, $\mathrm{Si}$ is the weight of branch $\mathrm{i}$, I's value ranges 1 - -M, after re-allocation of weight value, the weight value of branch $i$ is:

$$
S_{i}^{\prime}=S_{i}+\sum_{j=k}^{m} S_{j} \frac{S_{i}}{\sum_{i=1}^{n} S_{i}}
$$

Develop new teaching objectives after teaching evaluation, use "pruning" strategy generated educational objectives, that is dynamically generated node package weights, where Si 'is new weights of the i-th knowledge point, redistributed weights refer to, on the basis of the knowledge point's original weights, redistribute the cut knowledge points' weights to this knowledge point according to the proporation of each remaining knowledge points.

\section{Application of teaching evaluation in network teaching platform}

The System uses multi-angle evaluation criteria, to make a more comprehensive evaluation of learning subjects, it can not only evaluate learning with objective criteria, but also fully and comprehensively reflect learning ability of learning subjects, providing help for future learning. Meanwhile, it can provide guidance and constructive suggestions for teaching according to students' knowledge mastery situation.

In intelligent evaluation system, knowledge representation is the key to achieve system intelligence. Course knowledge of each chapter is an organic net structure system, knowledge points interrelate and interact with each other, making disciplinary network form a complete system of life. 
Represent discipline knowledge as knowledge tree, the increase of learned knowledge can be expressed as tree planting and growth process, which is a dynamic process.

This method can also be extended to students learning of multi-disciplinary knowledge, and multidisciplinary knowledge trees constitute the knowledge tree forest. Each specific subject's knowledge is subdivided according to knowledge granularity, knowledge tree internal nodes correspond to unit, chapter, section, subsection, and so on. Most courses have many knowledge points, in the previous study, students pay more attention to grasp of knowledge points rather than the intrinsic link between knowledge points, so learning subjects demonstrate poor flexibility and knowledge transfer capacity when doing comprehensive exercise, make more mistakes, without creatively study and apply. Cause of this problem is that students fail to establish the intrinsic link between knowledge points in the whole learning process, to form complete knowledge network. From another perspective, teaching evaluation contains cross-sections associated networking knowledge, which helps repeated knowledge memories and knowledge accumulation in the learning process.

\section{Summary}

Application of intelligent evaluation system, according to the study subject's own situation, provide appropriate guidance to their learning direction and content, so that they know exactly what to learn and how to learn during evaluation, do not waste time to repeat the exercise of mastered knowledge, through effective exercises and tests to achieve learning objectives, which greatly increases human color in the learning context, has important significance to improve learners' interest in learning, improve teaching effect.

This paper analyzes advantages and disadvantages of knowledge representation in traditional intelligent network teaching platform, based on this, proposes tree-based network hybrid knowledge representation and introduces tree network knowledge representation construction methods, the knowledge representation-based teaching evaluation contains various content of knowledge point, make comprehensive analysis of learning subjects and their learning process, and ultimately to develop individualized teaching objectives according to the different subjects.

\section{References}

[1] Zhu Huaqing, Cheng Yue and Hong Yan -The Application on Traversal of Knowledge Tree in Professional Courses Control [J] and China Education of Light Industry , 2010:53-55.

[2] Jiang Zuo and Yang Fan - Design and Realization of Knowledge Tree for Web Document [J] and Journal of Yunnan Nationalities University 2012,21(2):145-149.

[3] Nian Zhigang, Liang Shi, Ma Fanglan, et al. - Study and Application of Knowledge Expression [J] and Application Research of Computers, 2007(5):234-236,286.

[4] Liu Hongwei and Yao Shouguang - Application of Database Technique in Expert System Knowledge Representation [J] and Journal of Jiangsu University of Science and Technology (Natural Science Edition) 2007(6):50-53.

[5] Zeng Jianxun Knowledge way to build links to research [J]. Library and Information Service. 2010 (12) 